\title{
Prevention of infection in nurseries for the newborn
}

\author{
A. J. KEAY \\ M.B., Ch.B., F.R.C.P.(Ed.) \\ R. McD. SIMPSON \\ M.B., Ch.B.(Ed.)
}

Medical Paediatric Department, Western General Hospital, Edinburgh

\begin{abstract}
Summary
Prevention of infection in nurseries involves consideration of the design of hospitals, wards and equipment, the study of nursing and domestic procedures, the monitoring of environmental flora and the planning of antiseptic and antibiotic policies. These subjects cannot be considered in isolation from each other and are most suitably managed by a Control of Infection Committee. Ultimately the safety of infants in nurseries rests upon the degree to which each individual involved in their care pays attention to the agreed policies of general and personal hygiene.
\end{abstract}

STUDIES of perinatal mortality and morbidity such as those carried out by the National Birthday Trust (Butler and Alberman, 1969), have shown that many deaths and serious illnesses of the neonate are preventable. This has been confirmed by the improving figures for perinatal mortality and morbidity from this and other countries (World Health Organization, 1970). The figures for the U.K., however, lag behind those of other countries and this country appears to be moving downward rather than upward in the table. Many of the adverse factors associated with perinatal problems are socioeconomic in origin. In this and several other countries, there is a rising incidence of hospital delivery of pregnant mothers. In most areas of the U.K. over $95 \%$ of deliveries now take place in hospital. This hopefully beneficial arrangement may however place the infant at risk of nosocomial infection. A child's first exposure to the extra-uterine environment takes place in a hospital setting in which one should be capable of controlling the possibility of acquiring infection. Postnatally-acquired infection should be preventable. That it is not always prevented is a reflection on the clinical and bacteriological management of nurseries for the newborn. This paper considers some of the basic principles involved in the prevention of infection in nurseries for the newborn. The acquisition of organisms of any type by a newborn infant gives rise to changed circumstances but need not be harmful, and in certain situations may be beneficial. This is generally termed colonization and is superficial and confined to surfaces whether external, as on the skin, or internal, as in the gut. The immunological defences of the newborn against such invading organisms may be incomplete.

Where the combination of number or virulence of the invading organisms exceeds the infant's ability to limit the spread of those organisms, true infection will take place. This may remain superficial and such infections are not generally an immediate risk to the life of that infant. Should the infecting organism not be localized, spread to other tissues may occur and lead to illness and possibly death. Such infections may be termed deep, to distinguish them from the commoner but less serious superficial infections.

Although superficial infections are less serious for the individual infant they may prove a greater risk to other infants in the nursery. Thus the concept of infection in nurseries has to be considered not only in respect of the individual but also in relation to the group in which that individual is being nursed. Measures for the prevention of infection in the nurseries must be planned in respect of both the group and the individual infant in the nursery. This implies that in general what is necessary for any individual infant's protection against infection will be necessary for all other infants in the same group. Control measures must therefore be governed by policy decisions affecting a considerable number of medical, paramedical and non-medical personnel.

The simplest and most readily attainable objective of such a policy is the limitation of colonization of individual infants within a nursery so that there is no clinical infection in any infant in that nursery. This objective in turn implies control of the sources of the colonizing organisms. Although infection manifest in the newborn period may have been acquired antenatally or during passage down the birth canal, consideration will be given now to those infections which are acquired post-natally, and are generally accepted as nosocomial infections.

During the 1950s the ability to identify types of 
organism such às Staphylococcus aureus by phagetyping helped to clarify the sources of such organisms. In nurseries, the infants themselves and their immediate environment were shown to be the depots from which organisms came. The air and the hands of the medical and nursing staff were the principal methods of carriage. Recognition of the circles of infection and reinfection (Fig. 1) which were occurring led to efforts to break the cycles at vulnerable points and form the basis for policies for the control of infection in nurseries (Davies, 1971; Minkowski and Fleurquin, 1976).

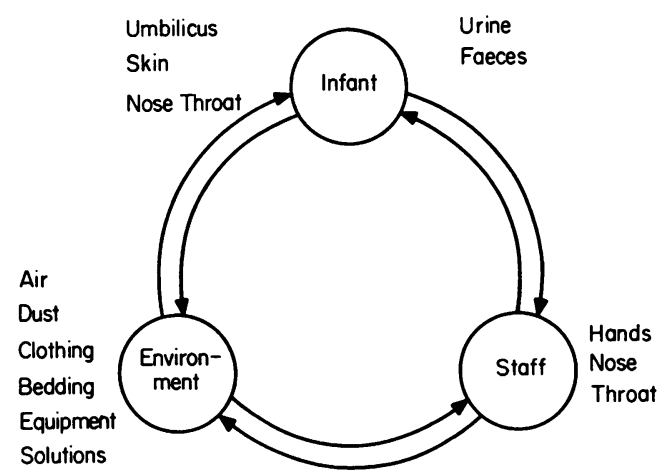

FIG. 1. Spread of organisms in nurseries.

Policy decisions have to be made in respect of hospital, ward and equipment, design and nursing procedures. On the basis of present knowledge of environmental flora, policies on the use of antiseptics and antibiotics can be formulated. The most fundamental policy decisions have to be made at a very early stage of hospital and ward design. Few practitioners, nowadays, have had the opportunity of taking part in the design of the hospital in which they work. Where such opportunity arises, experience does not suggest that they yet know all the answers to the problems concerned and the relative cost-effectiveness of expensive installations such as full air conditioning, laminar flow ventilation, etc., remain to be proved so far as general nurseries are recorded.

Design of individual wards and of alterations to existing ward spaces are more within the ambit of most clinicians and microbiologists. It is common experience that in any ward situation cross-infection increases with overcrowding. In other words, space is important. The Expert Group on Special Care for Babies (1971) recommended an allocation of a floor area of $4 \mathrm{~m}^{2} / \cot$ in rooms holding six to eight cots or incubators, and $5 \mathrm{~m}^{2} /$ cot in smaller rooms.

Experience in the Paediatric Department of the Western General Hospital, Edinburgh, has confirmed the value of these recommendations which are not, however, universally applied. Ready and rapid accessibility to each infant in a nursery is essential for dealing with emergencies and this may conflict with the concept of isolation as being beneficial for the limitation of infection. In special care nurseries, shortage of expert nursing staff often necessitates several infants being cared for by one nurse at a time, and wards for two to six cots or incubators are generally recommended as a useful compromise.

Special allocation of nursing staff to small wards should render possible isolation of that group of infants from other similar groups on the basis of 'cohort nursing'. The requirement for open visibility between the wards in neonatal nurseries limits the choice of materials available. Glass is generally an easy surface to keep clean although problems may arise where it is supported at its edges. Smooth surfaces with rounded corners and lack of dust traps are as important in the prevention of cross-infection as are the techniques of cleaning involved. Such requirements apply in greater measure to the incubator, the micro-environment in which many of the most infection-prone infants are nursed. Apart from their function in the regulation of temperature, incubators are also commonly used as sources of extra humidity from water tanks or by the use of nebulizers.

Gram-negative organisms frequently contaminat the reservoirs, tubing and apparatus involved, anc the design of such equipment should allow for easy cleaning and decontamination. If not adequately supervised, incubators, humidifiers, ventilators, suction apparatus and other such equipment used in special-care nurseries can do as much harm as good by acting as sources of infection or reinfection.

The use of disposable equipment has helped to reduce the incidence of cross-infection at a justifiable cost. Apart from the infants' mothers, midwives and nurses are those most frequently involved in the handling of infants, particularly those in the intensive or special care nurseries. Mothers may be carriers of Salmonella group organisms without being aware of it, and thus provide a source of infection which can prove very serious to the infants in a nursery for the newborn. Where such infections are endemic, screening of mothers by culture of stools becomes justifiable.

Nursing and domestic procedures within nurseries are of major significance as far as policies regarding prevention of infection are concerned. All such procedures should be aimed at reducing the contact of an infant with common objects such as bedding, feeding equipment, weighing apparatus, etc., which have been used for other infants. Contact with the hands of staff can be further reduced by nursing procedures such as keeping the infant in the same 
room as its mother. Where this is not possible, common changing, weighing or bathing rooms should be avoided and infants should be 'cot' nursed, so that as many nursing procedures as possible are carried out in the infant's own environment. When there is suspicion that infection may be present in a nursery, cohort nursing will isolate earlier batches of infants from later admissions. Barrier nursing is justifiable only in the management of infected infants. For routine nursing of healthy newborn infants there appears to be no advantage to be gained from time-consuming procedures such as masking and gowning (Forfar and Maccabe, 1958; Williams and Oliver, 1969; Evans, Akpata and Baki, 1971).

Domestic procedures, such as mopping and dusting, are as important as nursing procedures, and domestic personnel should be involved in discussions on prevention of infection and they should be educated in the importance of their role in this respect.

For all medical, nursing, and domestic staff as well as for the mothers, the overriding importance and value of the washing of hands must be rigidly stressed. Although work in the authors' department suggested that the air in the nurseries was a more important depot of infection than the hands of the attendant staff (Winton and Keay, 1968), it is widely accepted that the washing of hands between every handling of a patient and/or his excreta is of great importance in the reduction of infection in nurseries (Davies, 1971). This is best taught by the example of the senior staff whether medical, nursing or other in each unit.

Policy with regard to choice of antiseptic and antibiotic preparations to be used must be planned on a basis of knowledge of the environmental flora. This can only be achieved by collation of reports of the bacterial specimens taken for purely clinical reasons together with planned sampling of the environment for bacteria. The surfaces, solutions and equipment to be examined and the frequency with which examinations should be done should be decided by agreement of the staff concerned, and these agreements kept under review. High risk equipment, such as incubators, and particularly the water used for humidification purposes, should be sampled frequently, and the authors' department does this daily.

Patterns of environmental flora in nurseries have altered dramatically during the past 30 or $\mathbf{4 0}$ years. Before 1940 the impression is that haemolytic streptococci were the most important pathogens. During the 1940s and 1950s Staph. aureus was predominant; this was superseded in the 1960s by Gramnegative organisms - the water bugs. The 1970s have not yet evolved a clear-cut pattern but Franciosi,
Knostman and Zimmerman (1973), from their experience in the U.S.A., suggest that group B streptococcal infections may emerge as the most serious bacteriological problem. Figure 2 shows the organisms isolated from sampling the environment in the Special Care Nursery of the Western General Hospital, Edinburgh, and Fig. 3 shows those organisms cultured from the nineteen cases of infection encountered during 1975 . The patterns of isolates differ from those previously reported from this unit as shown in Fig. 4 (Keay, Syme and Barnes, 1967).

The incidence of clinical infection has fallen from the order of $10 \%$ of admissions in 1950 to $<2 \%$ in 1975. At the same time, the severity of the infections has declined and the only deaths occurring from infection in the past 5 years have been due to prenatally-acquired infection.

The advent of hexachlorophene coincided with a marked reduction in the incidence of staphylococcal infection in nurseries throughout the world (Simpson, Tozer and Gillespie, 1960).

Hyams, Counts and Monkus (1975) and Gehlbach et al. (1975) report recurrence of staphylococcal infection in nurseries where hexachlorophene has been withdrawn because of fears of its toxic effects. Others have shown that the reduction of staphylococcal infection has been bought at the price of a rise in Gram-negative infections (Forfar, Gould and Maccabe, 1968). Such infections have not necessarily diminished where hexachlorophene was withdrawn

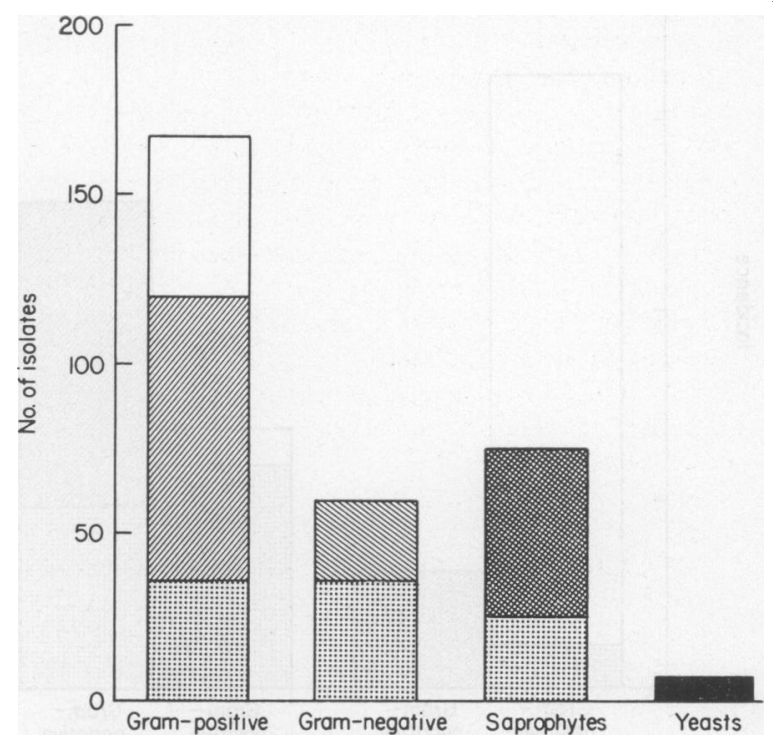

Fig. 2. Organisms isolated from the environment, Western General Hospital Nursery, 1975. $\square$ Staph. aureus; $\mathbb{Z}$, Staph. albus; $\mathbb{N}$, E. coli; Alkaligenes faecalis; $\square$, yeasts, 圈, others. 


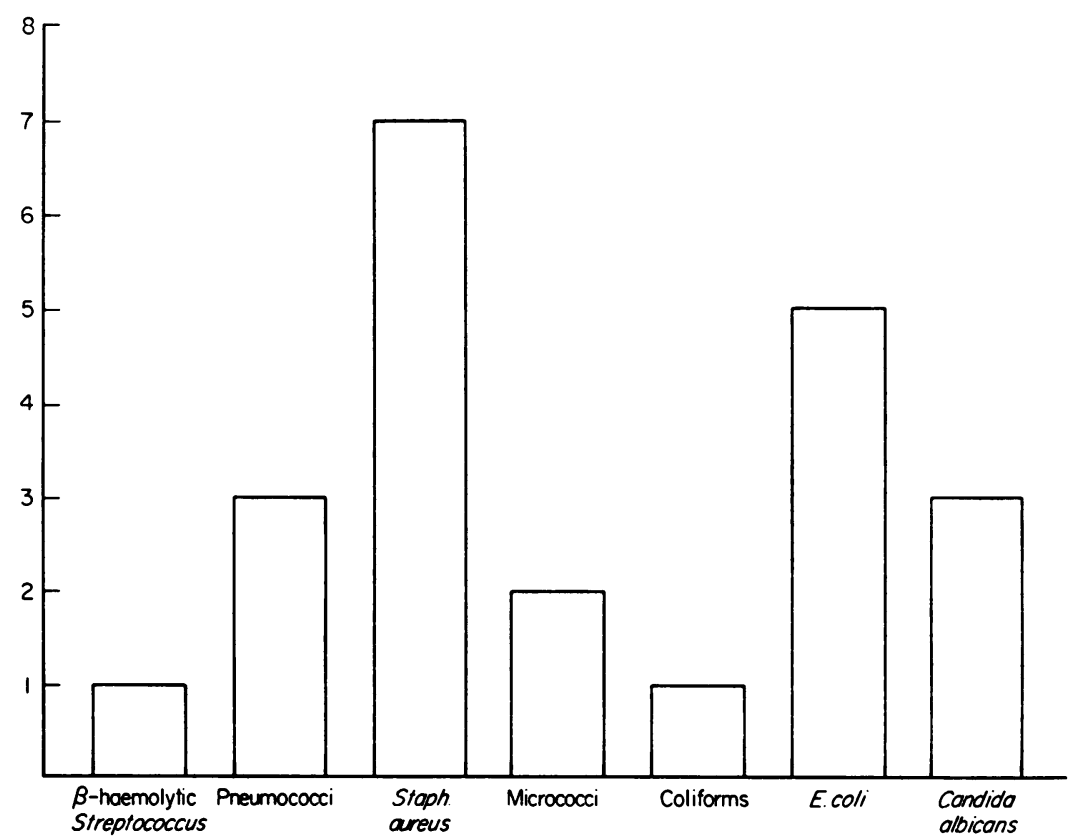

Fig. 3. Organisms isolated from clinical infections, Western General Hospital Nursery.

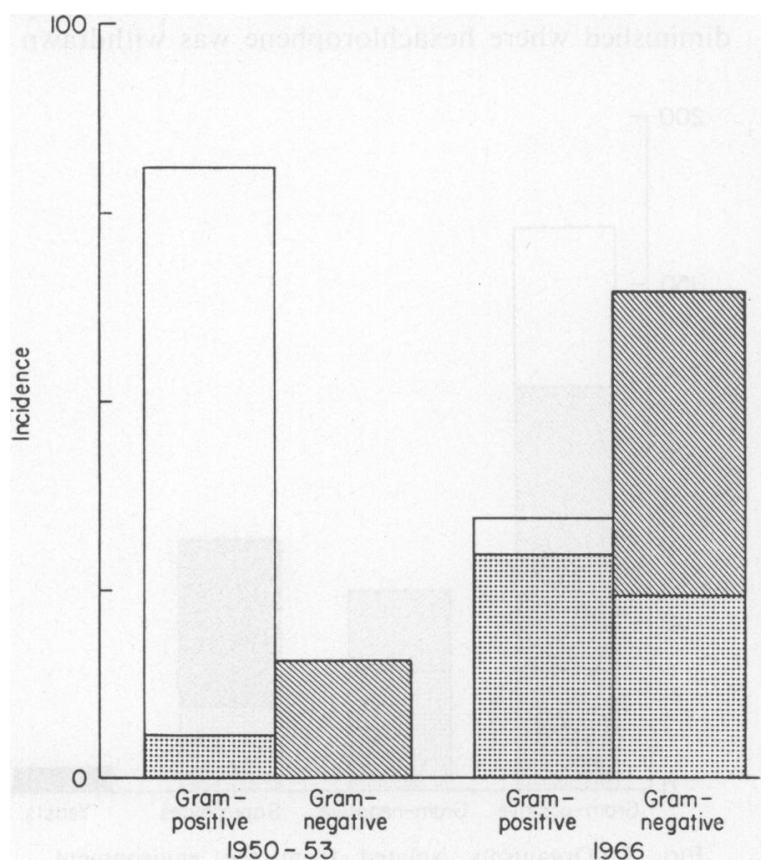

FIG. 4. Changing incidence of type of organisms, Staph. aureus; $\mathbb{N}$, E. coli; , others. nor have they increased where its controlled use was continued (Davies et al., 1970). Other antiseptics have been used in nurseries but none would appear to have had such a dramatic effect as the introduction of hexachlorophene. The type of antiseptic used should be governed by a policy decision taken by the appropriate Control of Infection Committee (Lowbury et al., 1975). The same committee should also be closely involved in consideration of antibiotic policy. This involves decisions on the circumstances in which antibiotics are to be used, and the choice of individual antibiotics (Keay et al., 1967). By limiting the use of antibiotics, by their careful selection and prescribed duration of treatment with them, the authors have reduced the use of antibiotics in their unit to what they believe to be a safe minimum. Prophylactic antibiotics should be used only in very particular situations which should be rigidly monitored.

Monitoring of the microbiology of the infants and the environment is the ultimate control of the efficiency of any method of prevention of infection. The extent to which this is done and its frequency have to be adjusted to suit local conditions but should never be completely omitted. Certain areas, such as intensive care nurseries, and certain equip- 
ment, such as incubators, require more frequent examination. Low birth-weight infants and those requiring artificial ventilation and intravenous or intra-arterial feeding are particularly at risk and require more intensive and more frequent monitoring of themselves and of their environment. By close co-operation between clinician and microbiologist any adverse change in environmental flora should lead to an immediate examination of that environment and the removal of the infants to a more suitable environment as soon as possible.

\section{References}

Butler, N.R. \& Alberman, E.V. (1969) Perinatal Problems. Second Report of the 1958 Perinatal Mortality Survey. Livingstone, Edinburgh.

DAvies, P.A. (1971) Bacterial infection in the fetus and newborn. Archives of Disease in Childhood, 46, 1.

Davies, P.A., Darell, J.H., Chandran, K.R. \& WaterWORTH, P.M. (1970) The efficacy of antibiotics in the neonatal period. In: Control of Chemotherapy (Ed. by P. J. Watt) p. 49. Livingstone, Edinburgh.

Evans, H.E., AkPata, S.O. \& BaHI, A. (1971) Bacteriologic and clinical evaluation of gowning in a premature nursery. Journal of Pediatrics, 78, 883.

Expert Group on SPECIAL Care for Babies (1971) Report of the Expert Group on Special Care for Babies. H.M. Stationery Office, London.

Forfar, J.O., Gould, J.C. \& MaCcabe, A.F. (1968) Effect of hexachlorophane on incidence of staphylococcal and Gram-negative infection in the newborn. Lancet, ii, 177.

Forfar, J.O. \& MACCABE, A.F. (1958) Masking and gowning in nurseries for the newborn infant. Effect on staphylo- coccal carriage and infection. British Medical Journal, 1, 76.

Franciosi, R.A., Knostman, J.D. \& Zimmerman, R.A. (1973) Group B streptococcal neonatal and infant infections. Journal of Pediatrics, 82, 707.

Gehlbach, S.H., Gutman, L.T., Wilfert, C.M., Brumley, G.W. \& KATZ, S.L. (1975) Recurrence of skin disease in a nursery: ineffectuality of hexachlorophane bathing. Pediatrics, 55, 422.

Hyams, P.J., Counts, G.W. \& Monkus, E. (1975) Staphylococcal bacteremia and hexachlorophane bathing. Epidemic in a newborn nursery. American Journal of Diseases of Children, 129, 595.

Keay, A.J., Syme, J. \& Barnes, P.M. (1967) Cephaloridine in the treatment and prophylaxis of infection in the newborn. Postgraduate Medical Journal, 43, (August Suppl.) 105.

Lowbury, E.J.L., Ayliffe, G.A.J., Geddes, A.M. \& Williams, J.D. (1975) Control of Hospital Infection. Chapman and Hall, London.

Minkowski, A. \& Fleurquin, N.P. (1976) Prevention of bacterial neonatal sepsis by contamination in an intensive care neonatal unit. Antibiotics Chemotherapy, 21, 121.

Simpson, K., Tozer, R.C. \& Gillespie, W.A. (1960) Prevention of staphylococcal sepsis in a maternity hospital by means of hexachlorophane. British Medical Journal, 1, 315.

Williams, C.P.S. \& Oliver, T.K. (1969) Nursery routines and staphylococcal colonization of the newborn. Pediatrics, 44, 640.

Winton, F.W. \& KeAY, A.J. (1968) Bacteria in a hospital nursery: laboratory and clinical studies. Journal of Hygiene, 66, 325.

World Health Organization (1970) The prevention of perinatal mortality and morbidity. WHO Technical Report Series, No. 457. 